\title{
Idiopathic pneumoperitoneum presenting as acute abdomen
}

\begin{abstract}
Aims: reporting a case of idiopathic pneumoperitoneum presented as an acute abdominal emergency with peritoneal signs,

Objectives: to discuss various aetiologies of pneumoperitoneum including non-surgical causes intrabdominal, intrathoracic, iatrogenic, rare case scenario of idiopathic spontaneous pneumoperitoneum and its management.

Case details: A 34 year old male presented with acute abdominal pain, vomiting and fever. Examination findings included diffuse abdominal tenderness, guarding and rigidity .leukocyte counts were normal, x-ray abdomen and chest showed air under both diaphragms. His medical history was unremarkable, no operative history or admissions previously. Patient was not on any medication, on smoker, non-alcoholic. Emergency laparotomy was performed no perforation of viscus was identified. Peritoneal wash was given and 3 drains placed. intraoperative peritoneal fluid sent for culture was sterile, patient subsequently recovered postoperatively and was stated orally on postoperative day 3 and was subsequently discharged on postoperative day 5 , the cause of pneumoperitoneum remained obscure.
\end{abstract}

Conclusion: a thorough history physical examination, appropriate lab tests and imaging are useful tools in identifying patients with non-surgical causes of pneumoperitoneum, Idiopathic pneumoperitoneum is rare and diagnosed only after a laparotomy.

Keywords: idiopathic pneumoperitoneum, idiopathic spontaneous pneumoperitoneum, surgical emergency
Volume 9 Issue 5 - 2019

\author{
Yashwant Singh Rathore, Raja Sumandatta \\ Aduri \\ Department of Surgical Disciplines, All India Institute of Medical
} Sciences, India

Correspondence: Yashwant Singh Rathore, Department of Surgical Disciplines, All India Institute of Medical Sciences, India, Tel +91991|337726, Email dryasvanth.r@gmail.com

Received: August 12, 2019 | Published: September 18, 2019

\begin{abstract}
Abbrevations: PP, pneumoperitoneum; IPP, idiopathic pneumoperitoneum

\section{Introduction}

Pneumoperitoneum (PP) is a radiologic term denoting an abnormal collection of air in the peritoneal cavity. Radiographic evidence of free intraperitoneal air is considered useful diagnostic tool for perforation of an intra-abdominal viscus.
\end{abstract}

About $85 \%-90 \%$ of patients with PP the cause is a ruptured intrabdominal viscus, most often from a perforated gastric or duodenal ulcer and other abdominal viscus PP without evidence of visceral perforation has been reported in $5 \%$ to $14 \%$ of all occurrences. ${ }^{1}$

Idiopathic pneumoperitoneum (IPP) is an even more rare condition from which both perforation of an intra-abdominal viscus and other known causes of free intraperitoneal gas have been excluded. We report a rare case, IPP presented with acute abdomen managed surgically

\section{Objectives: to report a rare case of IPP.}

to elucidate the various causes of PP and to emphasis on management of IPP.

\section{Case details}

34 year old male, presented to Emergency department with abdominal pain ,multiple episodes of non bilious vomiting and fever for 1 day duration, not passed flatus and stool since 2 days, history was unremarkable with no history of any Nonsteroidal anti-inflammatory medication intake, peptic ulcer disease, any operative procedure/ endoscopic procedures in the past .No history of Tuberculosis, Trauma, any past medical illness/admission, vital data:BP:106/60 PR:110/min, Respiratory rate: 20/min ,afebrile On examination distended ,umbilicus inverted central, diffuse abdominal tenderness, guarding and rigidity present, bowel sounds were absent.

\section{Investigations}

$\mathrm{X}$-ray erect abdomen and chest $\mathrm{x}$ ray revealed air under diaphragm (Figure1-2) .He underwent lab tests, which showed normal total leukocyte count $\left(8.67 \times 10^{\wedge} 3 / \mu 1\right)$, nevertheless his coagulation profile (prothrombin time: 20.2 INR: 1.85) was deranged suggestive of sepsis, peritoneal fluid(intraoperative) sent for culture and sensitivity was sterile.

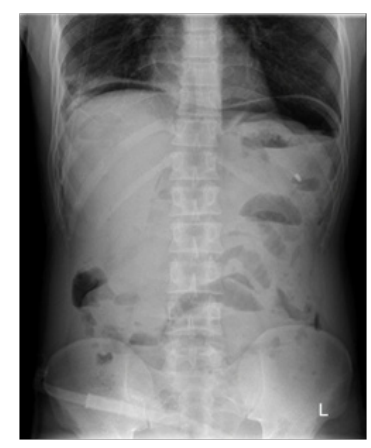

Figure I $\mathrm{X}$ ray erect abdomen. 


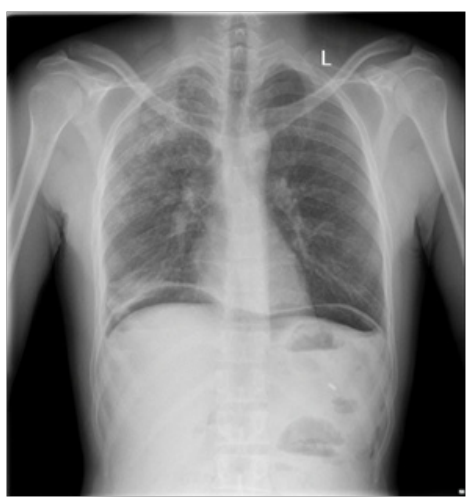

Figure $2 \times$ ray Chest PA view.

\section{Treatment}

Exploratory laparotomy was done, Non purulent infectious peritoneal fluid found, stomach, duodenum, and colon could be freely mobilized .No evidence of perforation was found in the distal oesophagus, stomach, duodenum, colon, or rectum, a passable stricture ileal stricture was identified, kocherisation was performed ,lesser sac was entered, colon \& caecum were mobilised, Air leak test negative (done by filling the abdomen with normal saline and injecting air through ng and rectum to detect gastric and rectal Tabel I various causes of PP perforation). Liver and gall bladder, spleen were normal. Warm saline wash given. 3 drains placed. Wound closed in layers. Skin left open (closed secondarily on 3rdpost-operative day).

\section{Post operative period and outcome}

Patient had uneventful recovery, received IV antibiotics and fluids, Did not have fever, total counts were normal, Started orally on postoperative day 3 and accepted oral feeds well. Drains had minimal output, right upper and left upper Drain was removed on day 3. Pelvic drain removed on post-operative day 4. Patient was discharged on post-operative day 5 . Follow up after 1 month patient was symptom free..$^{2-4}$

\section{Discussion}

By excluding perforation of the hollow viscera, and any other known cause of intraperitoneal free air, such as rupture of a diverticulum or trauma, a diagnosis of IPP is well established by exploratory laparotomy. This is consistent with previous reports of spontaneous PP. . $^{5,6}$

PP is a attributed to various causes including hollow viscus perforation, endoscopic or interventional procedures, thoracic causes, gynaecological, abdominal causes, post-operative, miscellaneous causes and rarely idiopathic (Table 1 ).

\begin{tabular}{lll}
\hline Common cause & Other causes & Rare \\
\hline & Thoracic: Intermittent positive pressure ventilation, \\
& Barotrauma, trauma, bronchoscopy, Cardiopulmonary \\
& resuscitation, Pulmonary Broncho pulmonary fistula, \\
& spontaneous rupture of pulmonary blebs & \\
& Abdominal: pneumatosis cystoides intestinalis, & \\
& spontaneous bacterial peritonitis endoscopic & \\
procedure & Distended hollow viscus, Emphysematouscholecystitis & Idiopathic(spontaneous) \\
perforation viscus & Ruptured hepatic abscess & \\
& jejunal diverticulosis, peritoneal dialysis & \\
& Gynaecological: vaginal insufflation, Pelvic \\
& inflammatory disease, coitus, vaginal douching \\
& Post-operative: retained air post open /laparoscopic \\
& procedures
\end{tabular}

In our patients, no definite cause was found, in spite of a thorough examination, so the aetiology remained "idiopathic". Mularski et al. in their systematic review, Among the 196 reported cases of spontaneous PP, 45 underwent surgical exploration without evidence of any perforation of the viscera. There are 20 cases of IPP have been published. Management of this condition is not standardized. PP occurs after open abdominal surgery in $60 \%$ or more of cases and is seen after laparoscopic procedures in about $25 \%$ of cases. postsurgical PP is by far the most frequent cause of nonsurgical PP.

Endoscopic gastrointestinal procedures also have been associated with the development of non surgical PP. Although PP after colonoscopy initially was thought to necessitate surgical exploration, conservative management is warranted in most cases, even if minor peritoneal signs are present. ${ }^{7}$ Pneumatosis cystoides intestinalis is the most common abdominal cause of nonsurgical PP that is not procedure related. It is characterized by multiple intramural gas- filled cysts, in any portion of the gastrointestinal tract but is most commonly found at the terminal ileum. Rupture of these submucosal and subserosal cysts causes PP. It is associated with collagen vascular diseases, bone marrow transplantation, malignancy, inflammatory bowel disease, acquired immunodeficiency syndrome. It generally resolves spontaneously, recurrence is well documented, management is non-surgical. ${ }^{1}$

Mechanical ventilation, trauma and cardiopulmonary resuscitation are associated with pneumothorax are also associated with PP. 3 main mechanism have been proposed for this occurrence

1) The direct passage through pleural and diaphragmatic defects. ${ }^{8}$

2) The passage of air from mediastinum via perivascular connective tissues to the retroperitoneum, entering the peritoneum through foramen of Winslow. ${ }^{1}$ 
3) Natural microscopic passage ways analogous to the proposed mechanism of cirrhotic fluid traversing the diaphragm. ${ }^{1}$

Gynaecological causes, though rare have been associated with non-surgical PP

Miscellaneous: scuba diving, adenotonsillectomy, and dental extraction ${ }^{2-4}$ have been reported to be associated with spontaneous PP on rare occasions. As with our cases no specific cause of perforation was found, it was labelled as IPP. PP with clinical signs and symptoms of acute abdomen warrant emergency laparotomy. In the absence of peritoneal signs with PP must remind surgeon of various non-surgical aetiology. However, in certain paediatric and immuno deficient patient peritoneal signs may present late, in case of suspicion exploratory laparotomy is warranted.

\section{Conclusion}

PP in an emergency setting is most commonly due to hollow viscus perforation, in the absence of peritoneal signs suspicion on nonsurgical causes should be kept in mind ,a thorough clinical history, examination and clinical discretion are required in making clinical decision in managing cases of idiopathic spontaneous PP.

\section{Learning points}

a. PP does not always mandate a surgical exploration

b. various non-surgical causes of PP should be remembered, when there is no ample clinical evidence of acute abdomen in a case with PP

c. Managment of spontaneous PP depends on the clinical condition of the patient, presence of peritoneal signs and other lab parameters, not all mandate surgical exploration

\section{Conflict of interest}

Author declares that there is no conflict of interest.

\section{Funding}

None.

\section{References}

1. Mularski RA, Sippel JM, Osborne ML. Pneumoperitoneum: a review of nonsurgical causes. Crit Care Med. 2000;28(7):2638-2644.

2. Rose DM, Jarczyk PA. Spontaneous pneumoperitoneum after scuba diving. JAMA. 1978;239(3):223.

3. Jash DK, An unusual complication during adenotonsillectomy. J Laryngo Otol. 1973;87(2):191-194.

4. Sandler CM, Libshitz HI, Marks G. Pneumoperitoneum, pneumomediastinum and pneumopericardium following dental extraction. Radiology.1975;115(3):539-540.

5. Karaman A, Demirbilek S, Akin M, et al. Does pneumoperitoneum always require laparotomy? Report of six cases and reviewof the literature. Pediatr Surg Int. 2005;21(10):819-824.

6. Omori $\mathrm{H}$, Asahi $\mathrm{H}$, Inoue $\mathrm{Y}$, et al. Pneumoperitoneum without perforation of the gastrointestinal tract. Dig Surg. 2003;20(4):334-338.

7. Taylor R, Weakley F, Sullivan B. Non-operative management of colonoscopic perforation with pneumoperitoneum. Gastrointest Endosc. 1978;24(3):124-125.

8. Mc Glone FB, Vivion CG, Meir L. Spontaneous pneumoperitoneum. Gastroenterology.1966;51(3):393-398.

\section{Acknowledgments}

None. 